\section{A real life clinical practice of neurologists in the ambulatory setting in Thailand: a pragmatic study}

\author{
Kannikar Kongbunkiat, 1 \\ Somsak Tiamkao, 1,2 \\ Verajit Chotmongkol, \\ Piyawan Chieawthanakul,3 \\ Sarawut Kitcharoen, 4 \\ Suthipun Jitpimolmard, 1 \\ Kittisak Sawanyawisuth ${ }^{5,6}$
}

1Division of Neurology, Department of

Medicine, Khon Kaen University;

2North-Eastern Stroke Research Group,

Khon Kaen University, ${ }^{3}$ Department of

Medicine, Khon Kaen Hospital;

4Department of Brain, Bangkok Hospital,

Rayong; ${ }^{5}$ Division of Ambulatory

Medicine, Department of Medicine, Khon

Kaen University; ${ }^{6}$ Research Center in

Back, Neck, Other Joint Pain and Human

Performance, Khon Kaen University,

Thailand

\section{Abstract}

The burden of neurological disorders is high in developing countries. Real life data from neurologists as to how they practice in Thailand are limited in literature. Practices of neurologists in a university hospital clinical setting in Thailand were studied. A prospective study was performed at the ambulatory neurology clinic, Khon Kaen University Hospital, between 1 February and 31 0ctober 2009. The following data were recorded: numbers of patients, characteristics of patients, consultation notes, and time spent for each patient. There were three neurologists, each of whom ran one afternoon clinic, once a week. There were 6137 visits during the 9 months, with an average of 681 visits per month. The total number of patients was 2834 . The three most common diseases were cerebrovascular diseases (33\%), epilepsy (16\%), and movement disorders (non-Parkinson's disease, 12\%). Neurologists spent an average of 6.34 minutes per patient. In conclusion, neurologists in medical schools have limited time to take care of each patient. Several strategies are needed in medical education and neurology training to improve the quality of care.

\section{Introduction}

A World Health Organization (WHO) report indicated that neurological disorders are a challenging problem in public health circles. Approximately 6.7 million patients died from stroke in 2012 worldwide and this ranked as the second leading cause of death after ischemic heart disease. ${ }^{1}$ Even in the US, there are several issues regarding the neurology workforce. ${ }^{2}$ The demand for neurologists is proposed to be 21,440 in the year 2025 with an approximate $19 \%$ shortfall resulting in long waiting times to see neurologists. In Asia and Europe the lack of neurologists is also problematic. ${ }^{3-6}$

In Thailand, the number of neurologists was also insufficient causing a heavy workload. The neurologists per population ratio in Thailand was 1:178,830, while the average ratio for Singapore was 1:81,132.7 Numbers of neurologists are needed to be increased worldwide to catch up with the increasing population. Not only is there the limited neurologist issue, a survey from Spain conducted in neurologists also showed the need to improve neurology training for both undergraduate and postgraduate physician training particularly for family practitioners. ${ }^{8}$

Approximately two thirds of neurological practices in Thailand were in the out-patient setting. ${ }^{9}$ To improve neurology training, knowledge of disease distribution and problems at the ambulatory neurology clinic is needed. The neurology training should focus on common neurological diseases as well as diagnoses of difficult diseases. The training issue also needs to deal with time constraints and limited educators. This study aimed to evaluate the functions and problems of the neurological clinic in a University Hospital in Thailand with the specific aim to improve quality of care and neurology training. The results here may apply to other tertiary care hospitals in Thailand as well as in Asian or Southeast Asian countries.

\section{Materials and Methods}

A prospective study was performed in the Ambulatory Neurological Clinic, Srinagarind Hospital, Khon Kaen University, Thailand. This healthcare facility is a university teaching hospital located in the northeast area of Thailand. The total number of patients for all disease possibilities is approximately 3000 outpatients per day.

The study period was between February 1 , and October 31, 2009. There were three neurologists providing services at the clinic, each one ran one afternoon session, once a week. Data were recorded on numbers of patients, characteristics of patients, consultation notes, and time spent for each patient. Data are presented by descriptive statistics. The study protocol was approved by the ethics committee of human research, Khon Kaen University.
Correspondence: Kittisak Sawanyawisuth, Department of Medicine, Faculty of Medicine, Khon Kaen University, 123 Mitraparp Road, Khon Kaen, 40002, Thailand.

Tel: +66.43 .363664 , Fax: +66.43 .348399 .

E-mail: kittisak@kku.ac.th

Key words: Ambulatory; neurological disorders; outpatient; pragmatic study; clinical practice.

Acknowledgements: the authors would like to thank the Mui Kiaw Sae Tae Research Fund in Neurology and the Faculty of Medicine, Khon Kaen University for grant support. We also would like to thank Prof. James A. Will (University of Wisconsin, USA) for his kind review of the manuscript.

Contributions: the authors contributed equally.

Conflict of interest: the authors declare no potential conflict of interest.

Received for publication: 30 January 2015 .

Revision received: 21 April 2015.

Accepted for publication: 27 April 2015.

This work is licensed under a Creative Commons Attribution NonCommercial 3.0 License (CC BYNC 3.0).

(O) Copyright K. Kongbunkiat et al., 2015 Licensee PAGEPress, Italy

Neurology International 2015; 7:5840

doi:10.4081/ni.2015.5840

\section{Results}

The total number of visits to doctors was 6137 in a period of 9 months (681 visits/month). The total number of patients was 2834. Of those, 1488 patients (53\%) were female, while 1346 patients (47\%) were male. Most patients were in the age range of 51-60 years (Figure 1). Most patients, 2652 patients or $93 \%$, were Thai, while 161 patients $(6 \%)$ were Chinese, 15 patients (1\%) were Laotian, and 6 patients $(0.2 \%)$ were Caucasians. The neurologists spent an average of 6.34 minutes per patient.

The ten leading diagnoses among the outpatients were: i) cerebrovascular diseases $(33 \%)$, ii) epilepsy $(16 \%)$, iii) movement disorders (MM, non-Parkinson's disease, 12\%), iv) Parkinson's disease (PD, 10\%), v) general medical diseases (4\%), vi) headaches (4\%), vii) neuromuscular junction (NMJ) diseases (4\%), viii) central nervous system (CNS) infection (3\%), ix) cranial nerve neuropathy (2\%), and $\mathrm{x}$ ) neurodegenerative diseases (2\%) as shown in Figure 2.

The patients were divided into 2 classes: 2317 former patients (82\%) and 517 new patients (18\%). Out of the 517 new patients, 
337 cases (65\%) had not been diagnosed before seeing a neurologist. Of these, 151 cases (29\%), however, had the correct diagnosis by the referring physician, whereas 29 patients (6\%) had wrong diagnoses. These patients with wrong diagnoses included myelopathy and radiculopathy, syncope, a spinal cord disorder, hydrocephalus, motor neuron disease, delirium, sleep disorder, polyneuropathy, tumors in the CNS, vertigo, neurodegenerative disease, neuropsychiatric disorder, peripheral nerve disorder, CNS infection, cranial nerve neuropathy, and muscle disease.

\section{Discussion and Conclusions}

Cerebrovascular disease was the most common disease in this study, similarly to WHO data. ${ }^{1}$ The rate of cerebrovascular disease was somewhat lower than the previous study in 1993 (33\% vs 38.4\%). With the time lapse of 16 years (1993 to 2009), the most commonly found disease remained the same. ${ }^{9}$ Note that numbers of patients with epilepsy and movement disorders except Parkinson disease were quite high. These findings were because there are epilepsy and botulinum toxin clinics in this hospital.

The main problem of neurological clinics in Thailand was the high numbers of patients. The survey in 1989 with 23 neurologists found that there were 1700 patients per week or 73.9 patients/neurologist/week. ${ }^{9}$ The present study showed that the average patients per neurologist per week was approximately 105 . This finding indicated that the numbers of patients seen by neurologists at the University Hospital were almost 1.5 times the 1989 average of Thailand. The average time spent per patient was only 6.34 minutes. During this time period, the neurologists needed to take care of patients and also supervise residents at the same time. The time limitation may affect the training of medical professionals. A survey performed by World Federation of Neurology found that only $65 \%$ of residents passed the national board examinations. ${ }^{6}$

Among the new patients, there were wrong diagnoses in only $6 \%$ of cases and $65 \%$ of new patients had no diagnosis prior to neurological consultation. In total, $71 \%$ of new patients were wrong- or undiagnosed. These patients were referred by general practitioners or internists. The diseases accounted for in this group were somewhat uncommon diseases such as radiculopathy, myelopathy, and neuropathy. Education and training in neurology for general practitioners and internists may improve this issue. Again, the problem will be part of the overwhelming issue of the workload for neurologists. The situation of difficulty in diagnoses of neurological diseases is also similar to Japan. ${ }^{3}$ Neurologists are required in general hospitals to assist the primary care doctors to diagnose rare neurological disorders.

Compared to other countries, the number of neurologists are fewer in both Thailand and the US, ${ }^{2}$ which causes long waiting times and short visits; only 6.34 minutes in this study in Thailand. University hospitals are the main postgraduate training sites for residents, $, 2,3,5$ but regional or general hospitals could play an important role in neurological training as well. This strategy may provide more experience for residents. Neurological training in Thailand occurs mainly at the university hospital as in most countries. ${ }^{2,3,5}$ Moving some training to the general or regional hospital may increase numbers of trained residents.

This study emphasized the need to improve

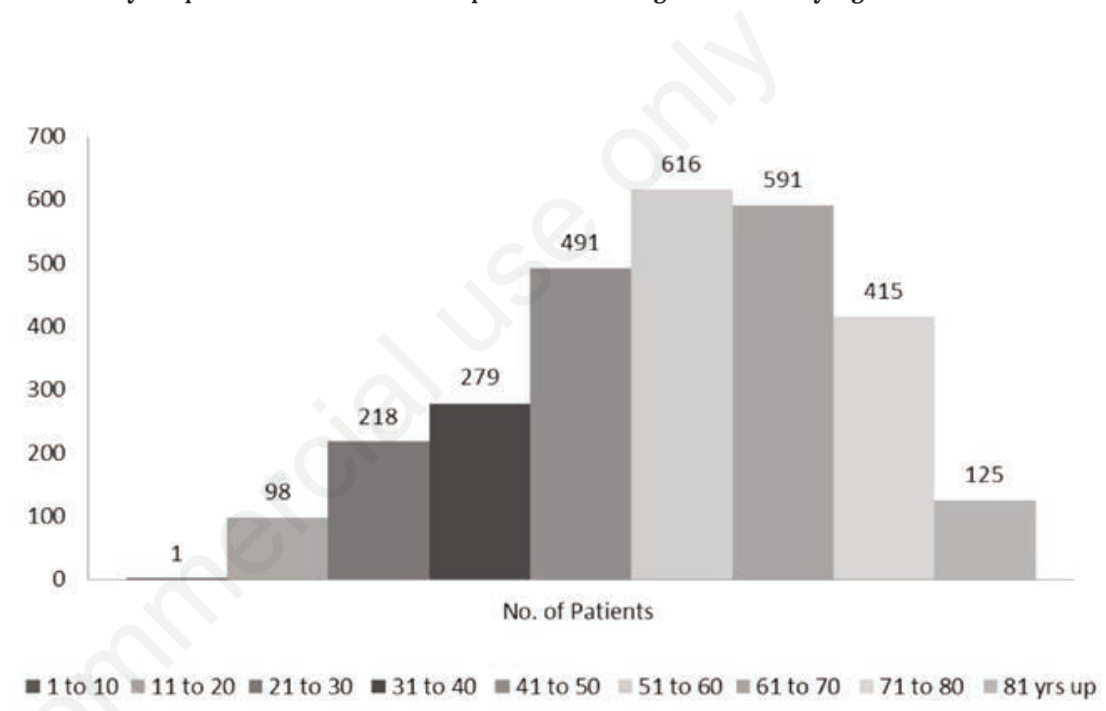

Figure 1. Age distribution of patients who attended the ambulatory neurology clinic during the study period.

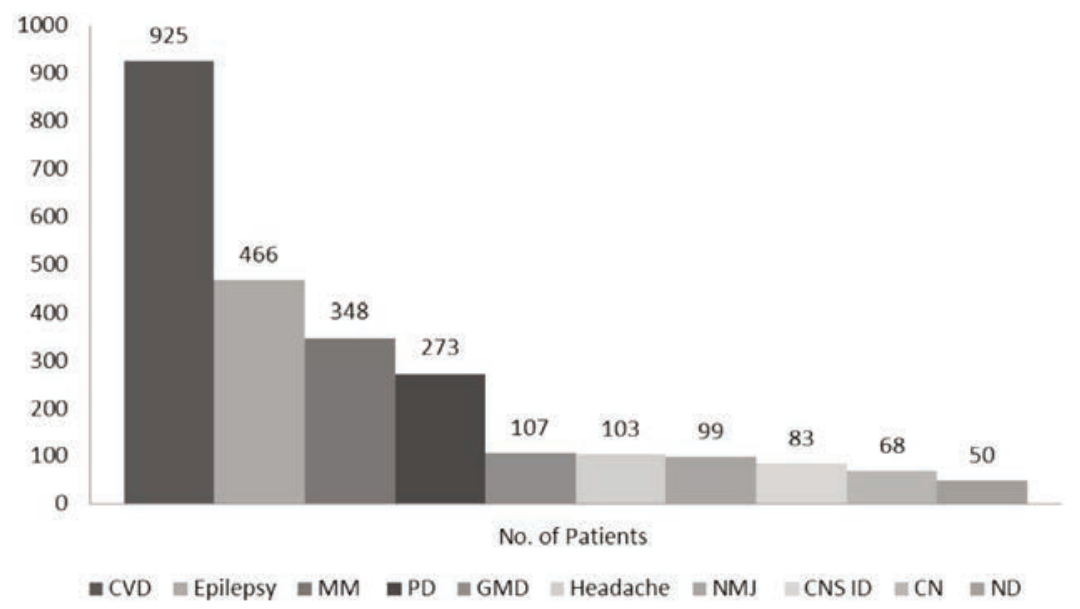

Figure 2. Disease distributions of patients who attended the ambulatory neurology clinic during the study period. CVD, cerebrovascular disease; MM, movement disorders; PD; Parkinson's disease; GMD, general medical diseases; NMJ, neuromuscular junction; CNS-ID, central nervous system infection; $\mathrm{CN}$, cranial nerve neuropathy; $\mathrm{ND}$, neurodegenerative diseases. ambulatory neurological care due to the high number of patients and limited numbers of neurologists. This survey also addressed workforce and workload issues of neurologists that are directly associated with burnout issues. The government should provide more support in funding training in neurology. With comprehensive training, general practitioners or ists should be able to handle simple neurological diseases including stroke patients and refer only difficult patients to neurologists. main limitation of this study is that it a study population that represented Another study should look at the outpatient clinical patient populations at regional and general hospitals. Improvement and additional training of general practitioners and internists at a higher level may lighten the load in terti- 
ary care hospitals in Thailand. In addition, a future study on inpatient workload of neurologists should be performed. This study included only the ambulatory workload because most neurological workload is located in the outpatient service. Evaluation of inpatient neurological services are also needed. Further collaborated studies with other Asian countries may provide insights into new strategies to lighten the load in tertiary hospitals and provide better training opportunities for residents, general practitioners and internists.

In conclusion, ambulatory neurology clinics are overwhelmed in university hospitals in Thailand. Neurology training focuses on common neurological diseases such as stroke, epilepsy, and movement disorders. Diagnostic training of other difficult diseases such as myelopathy or motor neuron disease should be emphasized as well.

\section{References}

1. WHO. The top 10 causes of death. Available from: http://www.who.int/mediacentre/factsheets/fs310/en/

2. Dall TM, Storm MV, Chakrabarti R, et al. Supply and demand analysis of the current and future US neurology workforce. Neurology 2013;81:470-8.

3. Yamane K. [Current status and needs of the department of neurology as a specialized department of a general hospital]. Rinsho Shinkeigaku 2000;40:1301-4. [Article in Japanese].

4. Benamer HT. Neurology expertise and postgraduate training programmes in the Arab world: a survey. Eur Neurol 2010;64:313-8.

5. Bereczki D, Csiba L, Komoly S, et al. [The carrier model of neurology in Hungary: a proposal for the solution until 2020]. Ideggyogy Sz 2011;64:377-84. [Article in Hungarian].

6. Bergen DC. World Federation of Neurology Task Force on neurological services: training and distribution of neurologists worldwide. J Neuro Sci 2002;198:3-7.

7. Poungvarin N. Resources and organization of neurology care in south east Asia. Neurology Asia 2007;12:41-6.

8. Bermejo-Pareja F. [Neurology and neurologists in Spain: the consolidation of the family neurologist?]. Neurologia 2007;22:221-35. [Article in Spanish]

9. Boongird P, Soranastaporn S, Menken M, Vejjajiva A. The practice of neurology in Thailand a different type of medical specialist. Arch Neurol 1993;50:311-2. 